\title{
Composite positive integers with an average prime factor
}

\author{
by
}

Florian Luca (Morelia) and Francesco Pappalardi (Roma)

Let $p(n)$ denote the average prime divisor of an integer $n$. That is,

$$
p(n)=\frac{1}{\omega(n)} \sum_{\substack{p \text { prime } \\ p \mid n}} p,
$$

where $\omega(n)$ denotes the number of distinct prime divisors of $n$.

It is clear that if $n$ is a prime power, then $p(n) \mid n$. In this paper we consider the set

$$
\mathcal{A}=\{n: \omega(n)>1, p(n) \in \mathbb{N}, p(n) \mid n \text { and } p(n) \text { is prime }\} .
$$

It is obvious that $n \in \mathcal{A}$ if and only if the square-free part of $n$ is in $\mathcal{A}$.

The first few square-free elements of $\mathcal{A}$ are: 105, 231, 627, 897, 935, 1365, 1581, 1729, 2465, 2967, 4123, 4301, 4715, 5313, 5487, 6045, 7293, 7685, 7881, 7917, 9717, 10707, 10965, 11339, 12597, 14637, 14993, 16377, 16445, 17353, 18753, 20213, 20757, 20915, 21045, 23779, 25327, 26331, 26765, 26961, 28101, 28497, 29341, 29607.

It is clear that $\mathcal{A}$ contains only odd numbers since otherwise $\omega(n)$ and $\sum_{p \mid n} p$ would have different parities and in order for $p(n)$ to be odd, $\omega(n)$ should be even and could not divide $\sum_{p \mid n} p$. Here, we prove the following result:

Theorem 1. Let $\mathcal{A}(x):=\mathcal{A} \cap[1, x]$. The estimates

$$
\begin{aligned}
\frac{x}{\exp ((2+o(1)) \sqrt{\log x \log \log x})} & \leq \# \mathcal{A}(x) \\
& \leq \frac{x}{\exp ((1 / \sqrt{2}+o(1)) \sqrt{\log x \log \log x})}
\end{aligned}
$$

hold as $x \rightarrow \infty$.

Since the counting function of the prime powers $n<x$ which are not primes is $O(\sqrt{x} / \log x)$, it follows that the same result is valid if we enlarge $\mathcal{A}$

2000 Mathematics Subject Classification: Primary 11N37; Secondary 11N60, 11P32. 
to be the set of all composite integers $n$ whose average prime factor is an integer and is a prime factor of $n$.

Our theorem complements the results from [1], where several results concerning the function $p(n)$ were obtained, such as the uniform distribution of the fractional parts $\{p(n)\}$ in the interval $[0,1)$ when $n$ ranges in the set of all positive integers, and the order of magnitude of the counting function of the set of positive integers $n$ such that $p(n)$ is an integer.

Throughout, we use the Vinogradov symbols $\gg$ and $\ll$ and the Landau symbols $O$ and $o$ with their regular meanings. We use log for the natural logarithm and \lfloor\rfloor for the "integer part" function.

Proof of the upper bound. Consider the following sets:

$$
\begin{aligned}
& \mathcal{A}_{1}(x)=\{n \leq x: P(n)<y\}, \\
& \mathcal{A}_{2}(x)=\left\{n \leq x: n \notin \mathcal{A}_{1}(x), P(n)^{2} \mid n\right\},
\end{aligned}
$$

where $y$ is a parameter which depends on $x$ to be chosen later and which satisfies $\exp \left((\log \log x)^{2}\right) \leq y \leq x$, and $P(n)$ denotes the largest prime factor of $n$.

From standard estimates for smooth numbers [2], we know that if we set $u=\log x / \log y$, then

$$
\# \mathcal{A}_{1}(x) \ll \frac{x}{\exp ((1+o(1)) u \log u)} \quad(x \rightarrow \infty)
$$

in our range of $y$ versus $x$, while

$$
\# \mathcal{A}_{2}(x) \leq \sum_{p \text { prime }}\left\lfloor\frac{x}{p^{2}}\right\rfloor \leq x \sum_{n \geq y} \frac{1}{n^{2}} \ll \frac{x}{y} .
$$

Let $\mathcal{A}_{3}(x)=\mathcal{A}(x) \backslash\left(\mathcal{A}_{1}(x) \cup \mathcal{A}_{2}(x)\right)$. If $n \in \mathcal{A}_{3}(x)$, then we can write $n=P(n) m$, where $m>1$ (because $\omega(n)>1$ ). Furthermore, since $n \notin$ $\mathcal{A}_{2}(x), P(n) \nmid m$, and $p(n)<P(n)$ since the average of at least two distinct integers is less than their maximum. Thus, the condition that $p(n)$ is prime and divides $n$ implies that $p(n) \mid m$, and so we can write

$$
p(n)=\frac{P(n)+\sum_{q \mid m} q}{\omega(m)+1}
$$

which gives

$$
P(n)=p(n)(\omega(m)+1)-\sum_{q \mid m} q .
$$

Hence, $P(n)$ is uniquely determined by $p(n)$ and by $m$. But since $p(n)$ is a prime divisor of $m$, it follows that for any fixed value of $m$, there are at most $\omega(m)$ possible values of $P(n)$. Furthermore, for the positive integers $n$ 
under consideration, we have $P(n) \geq y$, therefore $m \leq x / y$, so

$$
\# \mathcal{A}_{3}(x) \leq \sum_{m \leq x / y} \omega(m) \ll \frac{x \log \log x}{y},
$$

where we have used the well known fact that

$$
\sum_{t \leq x} \omega(t) \ll x \log \log x
$$

From (1)-(3), we immediately deduce that

$$
\begin{aligned}
\# \mathcal{A}(x) & \leq \# \mathcal{A}_{1}(x)+\# \mathcal{A}_{2}(x)+\# \mathcal{A}_{3}(x) \\
& \ll \frac{x \log \log x}{y}+\frac{x}{\exp ((1+o(1)) u \log u)} .
\end{aligned}
$$

To minimize the right hand side above we choose $y=\exp (u \log u)$, which amounts to

$$
\log ^{2} y=\log x \log \left(\frac{\log x}{\log y}\right) .
$$

Thus, we get $y=(1+o(1)) \sqrt{\log x \log \log x}$ as $x \rightarrow \infty$, and with this choice of $y$ versus $x$ we obtain

as $x \rightarrow \infty$.

$$
\# \mathcal{A}(x) \ll \frac{x}{\exp ((1 / \sqrt{2}+o(1)) \sqrt{\log x \log \log x})}
$$

Proof of the lower bound. Let $y$ be a parameter depending on $x$ (different from the one from the proof of the upper bound) and $k$ an even positive integer depending also on $x$, both tending to infinity with $x$ which we will choose later. For the moment we assume that $k>5$ and $y>k^{4}$. Suppose that $P, Q, p_{1}, \ldots, p_{k}$ are prime numbers which lie in the respective intervals:

$$
P \in(y / 2, y], \quad Q \in(y / 4, y / 2], \quad p_{1}, \ldots, p_{k} \in\left(y / 2 k^{2}, y / k^{2}\right] .
$$

It is clear that all the above primes are distinct and odd. Furthermore, the integer

$$
N=(k+4) Q-P-\left(p_{1}+\cdots+p_{k}\right)
$$

is odd, positive and

$N \geq k Q+4 Q-k \max \left\{p_{1}, \ldots, p_{k}\right\}>k y / 4+y-y-k\left(y / k^{2}\right)=k y / 4-y / k$, therefore it lies in the interval $(k y / 5, k y]$ once $x$ is sufficiently large. By Vinogradov's three primes theorem [3], the equation

$$
N=q_{1}+q_{2}+q_{3}
$$

has $\gg N^{2} / \log ^{3} N$ solutions in primes $q_{1}<q_{2}<q_{3}$ as $N \rightarrow \infty$. It is also clear that, at the cost of reducing the constant implied by the above $\gg$, we can assume that $q_{1}>c_{1} N$, where $c_{1}$ is some absolute positive constant, and that the three primes above are distinct. Note that with these choices, 
$\min \left\{q_{1}, q_{2}, q_{3}\right\}>c_{1} k y / 5>y$, therefore the primes $q_{1}, q_{2}$ and $q_{3}$ are different from $P, Q, p_{1}, \ldots, p_{k}$.

Consider the integer

$$
n=p_{1} \cdots p_{k} \cdot q_{1} \cdot q_{2} \cdot q_{3} \cdot P \cdot Q .
$$

We claim that $n \in \mathcal{A}$. Indeed, $\omega(n)=k+5$, and

$$
\frac{1}{k+5}\left(p_{1}+\cdots+p_{k}+q_{1}+q_{2}+q_{3}+P+Q\right)=Q
$$

is a prime factor of $n$. We are therefore only left with the task of counting the number of integers up to a fixed upper bound $x$ which can be constructed by the above method with suitable choices of $y$ and $k$ versus $x$.

For given $y$ and $k$, the number of choices for $P, Q$ and $\left(p_{1}, \ldots, p_{k}\right)$ are respectively:

$$
\pi(y)-\pi(y / 2), \quad \pi(y / 2)-\pi(y / 4) \quad \text { and } \quad\left(\begin{array}{c}
\pi\left(y / k^{2}\right)-\pi\left(y / 2 k^{2}\right) \\
k
\end{array}\right) .
$$

Therefore the number of possible $n$ 's, when $k^{4}<y$ and $k$ is large, is

$$
\gg \frac{y}{2 \log y} \cdot \frac{y}{4 \log y} \cdot\left(\frac{y}{6 k^{3} \log \left(y / k^{2}\right)}\right)^{k} \cdot \frac{c_{1}(k y / 4)^{2}}{(\log k y)^{3}},
$$

where in the above estimates we used the prime number theorem and the fact that if $a>2 b$, then

$$
\left(\begin{array}{l}
a \\
b
\end{array}\right) \gg\left(\frac{a-b}{b}\right)^{b}>\left(\frac{a}{2 b}\right)^{b}
$$

with the choices $a=\pi\left(y / k^{2}\right)-\pi\left(y / 2 k^{2}\right)>y /\left(3 k^{2} \log \left(y / k^{2}\right)\right)>2 k$ and $b=k$ (the first estimate above holds for large $k$ by the prime number theorem, while the second holds for large $k$ by the fact that $y>k^{4}$ ).

A further calculation shows that the expression appearing at (4) above is

$$
\gg \frac{y^{k+4}}{4^{k} k^{3 k-3}(\log y)^{k+5}} .
$$

We now need to find a lower bound on the above expression under the constraint that

$$
n=p_{1} \cdots p_{k} \cdot q_{1} \cdot q_{2} \cdot q_{3} \cdot P \cdot Q \leq\left(\frac{y}{k^{2}}\right)^{k}(k y)^{3} y^{2}=: x .
$$

We will do this by choosing $k=\lfloor c \sqrt{\log x / \log \log x}\rfloor+\nu$, where $\nu \in\{0,1\}$ is such that $k$ even and $c$ is a constant to be determined later. Then, by 
estimate (5), we get

$$
\begin{aligned}
\# \mathcal{A}(x) \geq & \frac{x}{\exp (k \log 4 k+\log y+(k+5) \log \log y)} \\
= & x \exp (-c / 2 \sqrt{\log x \log \log x}-\log y c \sqrt{\log x / \log \log x} \log \log y \\
& -O(k+\log \log y)) .
\end{aligned}
$$

Estimate (6) together with the choice of $k$ leads to the conclusion that $\log y=c^{-1}(1+o(1)) \sqrt{\log x \log \log x}$ as $x \rightarrow \infty$, which, in turn, leads to the lower bound

$$
\# \mathcal{A}(x) \gg \frac{x}{\exp \left(\left(c+c^{-1}+o(1)\right) \sqrt{\log x \log \log x}\right)} .
$$

The minimum of the function $c \mapsto c+c^{-1}$ is attained at $c=1$. Hence, choosing $c=1$, we get the lower bound of the statement.

Acknowledgements. This work was done in February of 2006, while both authors were in residence at the Centre de Recherche Mathématique of the Université de Montréal for the thematic year Analysis and Number Theory. The authors thank the organizers for the opportunity of participating in this program.

\section{References}

[1] W. D. Banks, M. Z. Garaev, F. Luca and I. E. Shparlinski, Uniform distribution of the fractional part of the average prime factor, Forum Math. 17 (2005), 885-903.

[2] A. Hildebrand, On the number of positive integers $\leq x$ and free of prime factors $>y$, J. Number Theory 22 (1986), 289-307.

[3] I. M. Vinogradov, Representation of an odd number as a sum of three primes, C. R. (Doklady) Acad. Sci. USSR 15 (1937), 291-294 (in Russian).

Instituto de Matemáticas

Universidad Nacional Autónoma de México

C.P. 58089

Morelia, Michoacán, México

E-mail: fluca@matmor.unam.mx
Dipartimento di Matematica Università Roma Tre Largo S. L. Murialdo 1 00146 Roma, Italy E-mail: pappa@mat.uniroma3.it

Received on 4.1.2007

and in revised form on 30.4.2007 